\title{
Fault Causes Analysis in Feeders of Power Distribution Networks
}

\author{
O. A. Quiroga, J. Meléndez, S. Herraiz \\ Institute of Informatics and Applications, University of Girona \\ Campus Montilivi - Av. Lluis Santaló, 17071 Girona (Spain) \\ Phone/Fax number: +0034972 418486, e-mail: \{oscar.quiroga, joaquim.melendez, sergio.herraiz\}@udg.edu
}

\begin{abstract}
In this paper, a basic statistical analysis about principal causes of faults in power distribution networks is presented. Principal failure causes are identified through basic statistical and PCA (Principal Component Analysis) is used to find combinations of causes or other factors that describe major trends in the data set in terms of data variability.

The idea is to exploit information available related with the causes of faults occurred over an important period of time in a real power network. Common causes, trends as well as relations between causes and feeders types (overhead and underground) are found. Relevant information to know the behaviour of faults in a network is extracted.
\end{abstract}

\section{Key words}

Failure causes, fault, incidence, voltage sag, PCA.

\section{Introduction}

The failure of components in power networks can cause problems related with power quality and reliability [1]. The solutions of such problems are of interest both for the utilities and customers.

Factors such as environmental conditions, external agents or component status are involved in the occurrence of faults and failures. While faults are short circuits caused by dielectric breakdown of the insulation system, the failures are the termination of the ability of the components to perform its required functions [2]. The components failures normally induce faults and these faults are reflected as defects in the supply voltage. Utilities also use the term incidence to refer the actions implemented to return the systems to normal operation when faults and failures have occurred. Voltage sags are the main defect caused by faults in power networks.

Maintenance policies (preventive, based on condition monitoring, etc) are implemented to reduce the impact of the faults on the network. Also, fault prediction based on the early identification of symptoms, or incipient faults, leading to the appearance of faults is a topic of interest in power systems and several frameworks are available. Moreover, several methods have been developed to estimate the number and severity of faults in the network, based on the study of the voltage sags in a stochastic sense. However, methods must be developed to improve the systematic analysis of events caused by faults and their propagation along the network in order to discover fault patterns associated with frequent causes and their origin or to maintain accurate fault diagnoses and predictions.

The huge number and variety of components (overhead lines, cables, circuit breakers, transformers, fuses, insulators, etc.) that can be affected by failures makes difficult to deploy monitoring strategies that individually supervise all of these elements. So, new paradigms are needed to develop low cost solutions to maintenance policy difficulties. They have to be oriented to extract and exploit useful information from historical data and on-line events generated in both normal and abnormal conditions.

This work focuses on faults and failures generated in power systems and recorded in substations, and related information whit these faults (normally collected by the utility) such as cause, failed component, location, etc., with the aim of knowing the main causes of faults and their contribution on the global generation of incidences on the distribution network. Since not all the faults or incidences are produced by failures and these have different natures and causes, the goal is to recognize statistical information that shows the behaviour of the causes and the relation whit the lines topology.

The main idea is to exploit the information available related with the causes of faults occurred over an important period of time. Common causes, correlations between causes and feeders types (overhead and underground) can be found. According to [6] the information about the failure cause is of special importance since the consequences of failures can be prophesied and the appropriate measures of maintenance may be devised by evaluating it. 


\section{Faults, Failures and Incidences}

\section{A. Faults and Failures}

Faults are abnormal state of the network, usually associated with short circuits or insulation breakdown between two or more points, which cause the actuation of protective (automatic and manual) systems. Failure is the termination of the ability of a component or item to perform its required function.

Faults can be temporary or permanents. A temporary fault occur when the actuation of protective systems allowing the circuit to be re-energized (fault clearing) after a reclosing operation. Examples of temporary faults are the insulation breakdown by the interaction between components and external agents (lightning strikes, wind, transient tree contacts, etc.) during a short period of time. Permanent faults require to repair or to replace the damaged component. Examples of permanent faults include insulators damage by flashover, underground cable breakdown and surge arrester damage. This second type of faults shows the occurrence of a failure.

\section{B. Incidences}

Incidences make reference to situations in the network that requires an action to return to its normal operating condition, normally are related with faults and failures. An incidence can be related with one or several faults. Examples of incidences are a failure in a cable by aging or also the actuation of protective system in the head of a feeder by a lightning.

\section{Study Case}

The idea is to extract statistical information to understand the behaviour of the occurred faults in a power network. In this paper, information of incidences occurred over eight years in several feeders of a power distribution system is analyzed. The incidences were documented by the utility, that is, information related to the origin, causes and activation of protective systems is available.

\section{A. Network topology.}

The monitored distribution network is formed by underground and overhead feeders. In general, $30 \%$ of the network is composed by underground components and $70 \%$ by overhead components.

\section{B. Causes and main types of incidences.}

Failures, self-extinguishing faults with actuation of the header protective systems, and also fuse actuation in branches of the network are the main types of incidences. Environmental conditions (rain, lightning, wind, storms, etc.), external factors (birds, trees, etc.), internal factors (aging, self manoeuvres of the network, etc.) are the main causes of such incidences. Fig. 1 shows that about of $80 \%$ of the incidences are self-extinguishing faults and $20 \%$ are failures.

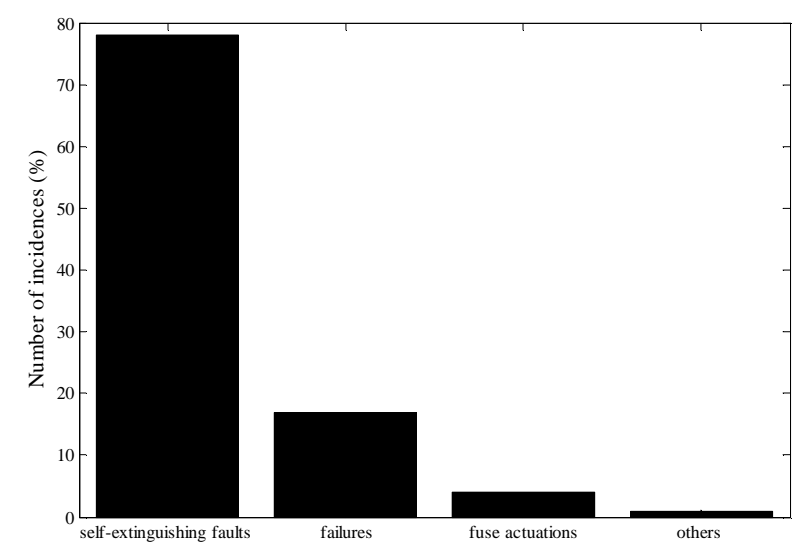

Fig. 1. Main types of incidences monitored in substations.

Fig. 2 shows the main causes of self-extinguishing faults, majority $(61 \%)$ are by unknown causes followed by environmental causes $(33 \%)$ such as storm, wind or rain. Other causes $(6 \%)$ are for example birds, or manoeuvre errors.

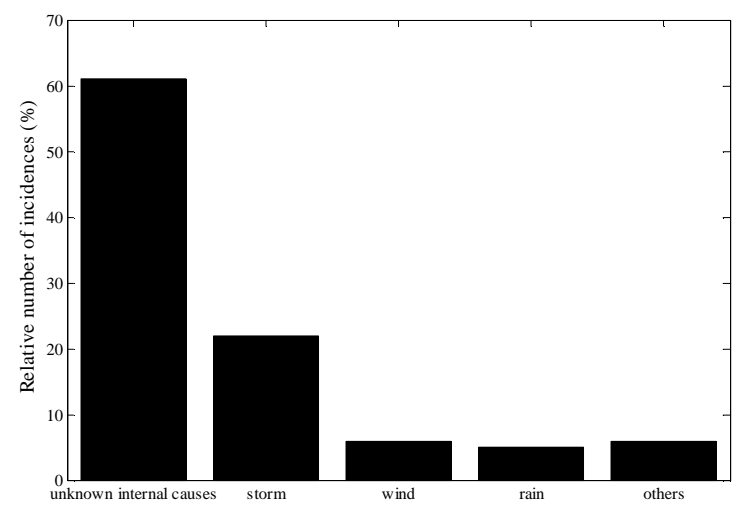

Fig. 2. Main causes of self-extinguishing faults.

Fig. 3 shows the main causes of failures. Aging (37\%) and environment causes $(32 \%)$ are the more important factor of failures. However, accidents (13\%) also represent an important factor of failures. This item includes, for example, damages in cables by excavation activities, cable contacts with vehicles and vandalism.

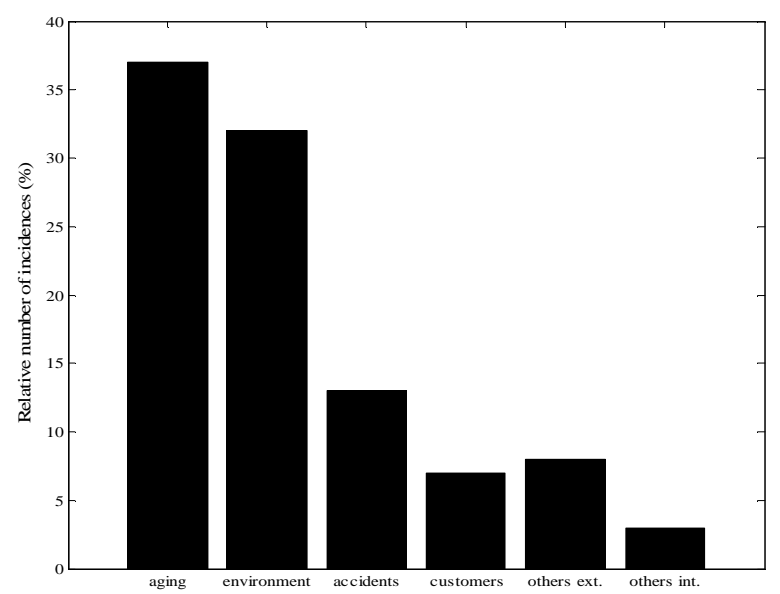

Fig. 3. Main causes of failures.

Results shown in Fig. 3 are consistent with [6], which exposes that in distribution networks the primary causes 
for failure are the aging (decrease of electrical strength, thermal strength and mechanical strength) and random failure (environmental causes, accidents, etc).

\section{Analysis of Incidences and Failures.}

Several maintenance policies are implemented to reduce the number of faults, failures and incidences on the network. Historical information is very important to assess the performance of maintenance measures implemented. Faults rate in a power network is related whit the supply reliability because if the number of faults increases the supply reliability probably decreases.

Fig. 4 shows the incidence and failure rates per $\mathrm{km}$ for several years according to the recorded information. The failure rates for two main causes of failures (aging and atmospheric influences) are also plotted. Incidence rate per $\mathrm{km}$ of line change year to year and don't follow a specific trend while failure rate and failure rate by aging have an increasing tendency. This tendency, especially in the failures by aging is likely consequence of the vegetative growth. Accidental occurrences (mainly by excavation activities) and environment influences are other reasons for increasing the failure rate.

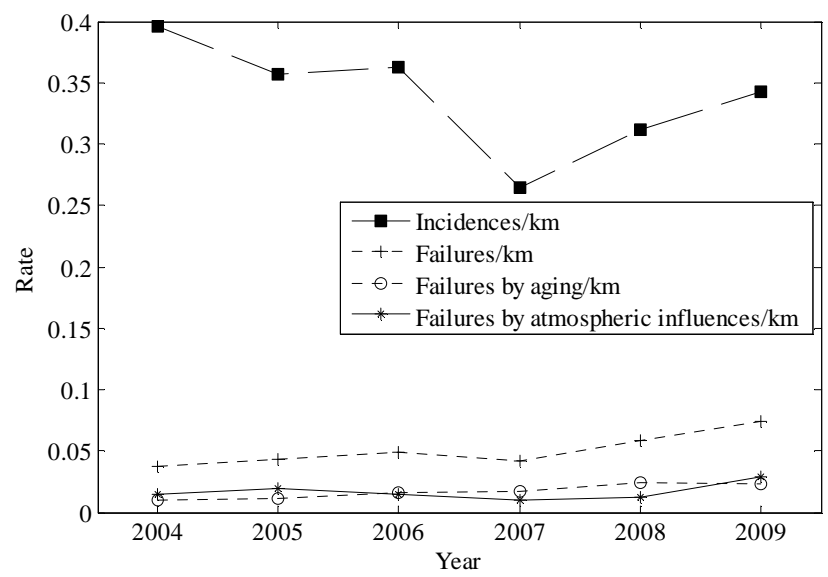

Fig. 4. Incidence and failure rates.

Incidences are caused by many external and internal factors. Aging is the most important factor of failures followed by atmospheric causes as Fig. 3 shows, but these factors have different effect on underground and overhead lines. Also the number of incidences depends on the length and type of line (underground and overhead). About $80 \%$ of incidences are self-extinguishing fault as was shown in Fig. 1 but the majority of these faults have their origin in aerial portions of the lines which are exposed to the environment.

While in underground lines failures are produced mainly by aging or damage in insulation materials (including accidents related with excavation activities), in overhead lines failures are caused by random causes such as atmospheric influence (storms, lightning, etc.), vegetation and animals. To test the correlations between the number of incidents, failures by aging, failures by atmospheric influences and lines length (overhead and underground)
KMO index (Kaiser-Meyer-Olkin measure of sampling adequacy) was used and a KMO value bigger than 0.7 was calculated. KMO [7] is an index for comparing the magnitudes of the observed correlation coefficients to the magnitudes of the partial correlation coefficients and it is used to measure the appropriateness of factor analysis. High values (between 0.5 and 1.0) indicate factor analysis is appropriate. Values below 0.5 imply that factor analysis may not be appropriate. Since the partial correlations between variables in the data set are important, a PCA (Principal Component Analysis) can be done to discover the trends between variables.

According to [8], PCA is a technique for data compression and information extraction. More concretely, PCA is used to find combinations of variables or factors that describe major trends in a data set in terms of data variability. According to PCA basis, the normalised dataset $X$ (rows, $n$, represent observations and columns, $m$, represent variables with zero mean and unit variance), can be expressed as a linear combination of $r$ new variables, assuming an error matrix $E$.

$$
X=\sum_{i=1}^{r} \overrightarrow{t_{l}} \times \overrightarrow{p_{l}^{T}}+E
$$

Where $\overrightarrow{t_{l}}$ and $\overrightarrow{p_{l}}$ are named scores and loading vectors respectively and are computed to reflect relevant relation among variables $\left(\overrightarrow{t_{l}}\right)$ and observations $\left(\overrightarrow{p_{l}}\right)$. PCA assumes that the loadings associated to the biggest eigenvalues of the covariance matrix of dataset represent the best directions for expressing the data, upon based on the maximum variance criteria. By retaining only a few of them, it is possible to represent the majority of the variance of the original data set. Not retained information, represented by $E$, is associated with noise and less meaningful variations. Thus, the first $r$ principal components, instead of the $m$ original variables, build up a new space/model with a lower dimensionality than the original one.

For the PCA, the observations are the distinct monitored feeders and the variables are: number of incidences (INCIDENCES), number of failures by aging (AGING), number of failures by atmospheric influences (ENVIRONMENT), length overhead in $\mathrm{km}$ (OVERHEAD) and length underground in $\mathrm{km}$ (UNDERGROUND). The first two components explain the $72.93 \%$ of the dataset variability $(49.73 \%$ and $23.21 \%$ by the first and the second component, respectively). Results are summarized in Fig. 5. In this figure, the original variables are shown as a function of these two principal components and the relations between each variable can be seen most clearly than in the original space.

According to Fig. 5 there are mainly two groups in the observations. First group is composed by feeders whose variables OVERHEAD, INCIDENCES and ENVIRONMENT are dominants while the second group is characterized by the variables UNDERGROUND and AGING. This means that aging is the main cause of 
failures in underground lines, and atmospheric influences is the first cause of failures in overhead lines. In overhead lines the number of recorded incidences is also greater than in underground lines.

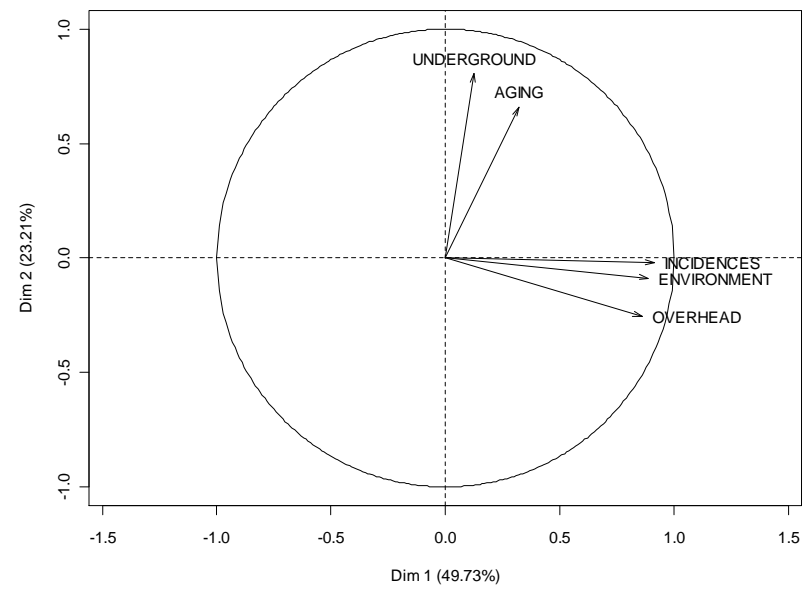

Fig. 5. Main causes of incidences and types of lines in PCA space.

\section{Conclusions}

In this paper, we explore the main causes of incidences and failures in an electric distribution networks based on information monitored along of several years. Whit this information some indicators such as the incidence rate, failure rate, failure rate by aging and failure rate by atmospheric influences was obtained per $\mathrm{km}$ of line. Result show that year to year the number of incidences rate do not follow a specific trend whereas the failures follow an increasing trend.

In overhead feeders arise the higher number and causes of incidences. These feeders are longer than underground feeders and are exposed to the environment. While aging is the main cause of failures in underground feeders, atmospheric influences are the most important in overhead feeders and they are mainly derived from mechanical damage in the conductors.

Recording and analysis of the information related with the causes, frequency and origin of faults and failures are very important to know the behaviour of the faults and support the maintenance activities but information collected along of many years is required.

\section{Acknowledgement}

The work has been supported by the research project "Monitorización Inteligente de la Calidad de la Energía Eléctrica" (DPI2009-07891) funded by Ministerio de Ciencia e Innovación (Spain). It has been developed within the eXiT, Control Engineering and Intelligent Systems, research group of the Institute of Informatics and Applications (University of Girona). eXiT is part of the Automation Engineering and Decision Support Systems (AEDS) research group, awarded with a consolidated distinction (2009 SGR 523) for the 2009-2012 period in the Consolidated Research Group (SGR) project of the Generalitat de Catalunya.

Oscar Quiroga received a $\mathrm{PhD}$ scholarship (2009FIA00452) funded by the Comissionat per a Universitats $i$ Recerca del Departament d'Innovacio, Universitats $i$ Empresa of the Generalitat de Catalunya and also the European Social Fund.

The authors thank the utility Endesa Distribución for data and information support.

\section{References}

[1] M. Bollen, Understanding Power Quality Problems, Voltage Sags and Interruptions, IEEE press series on power engineering, Piscataway USA (1999).

[2] G. Olguin, "Voltage dip (sag) estimation in power systems based on stochastic assessment and optimal monitoring," Ph.D. dissertation, Dept. Energy Environ., Div. Electr. Power Eng., Chalmers Univ. Technol., Goteborg, Sweden, 2005.

[3] R. Agrawal and R. Srikant. "Mining sequential patterns," International Conference on Data Engineering ICDE'95, pp. 3-14.

[4] H. Mannila, H. Taitoven and A. I. Verkamo, "Discovery of Frequent Episodes in Event Sequences", Data Mining in Knowledge Discovery, vol. 1, no. 3, pp. 259-289, Sep. 1997.

[5] Z. Liao, G. Wang, Q. Ye, and Y. Sun, "A novel fault diagnosis system for transmission line system based on sequence of events," in Advances in Power System Control, Operation and Management, 2003. ASDCOM 2003. Sixth International Conference on (Conf. Publ. No. 497), vol. 1, 11-14 Nov. 2003, pp. 440-445.

[6] X. Zhang and E. Gockenbach, "Component reliability modeling of distribution systems based on the evaluation of failure statistics," Dielectrics and Electrical Insulation, IEEE Transactions on, vol. 14, no. 5, pp. $1183-1191$, Oct. 2007.

[7] A. P. Field, Discovering Statistics Using SPSS. London: Sage (2005).

[8] X. Berjaga, Á. Pallarés and J. Meléndez, "A Framework for Case-Based Diagnosis of Batch Processes in the Principal Components Space," Emerging Technologies \& Factory Automation 2009. ETFA 2009. IEEE Conference on, pp. 1, 22-25 Sept. 2009. 